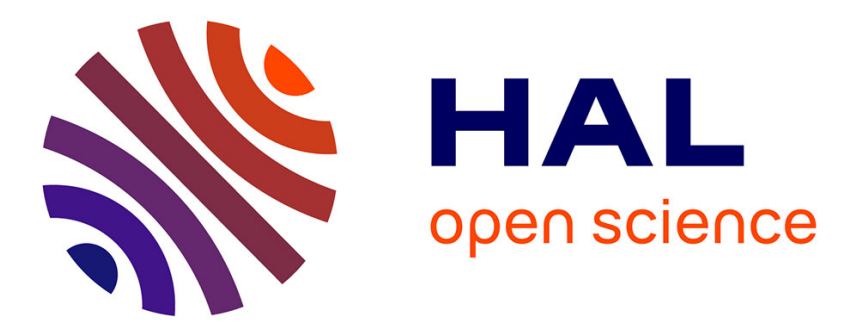

\title{
Influence des régions de charge d'espace superficielles sur l'absorption optique des couches minces polycristallines de Zns
}

P. Bugnet

\section{- To cite this version:}

P. Bugnet. Influence des régions de charge d'espace superficielles sur l'absorption optique des couches minces polycristallines de Zns. Revue de Physique Appliquée, 1974, 9 (2), pp.447-450. 10.1051/rphysap:0197400902044700 . jpa-00243800

\section{HAL Id: jpa-00243800 https://hal.science/jpa-00243800}

Submitted on 1 Jan 1974

HAL is a multi-disciplinary open access archive for the deposit and dissemination of scientific research documents, whether they are published or not. The documents may come from teaching and research institutions in France or abroad, or from public or private research centers.
L'archive ouverte pluridisciplinaire HAL, est destinée au dépôt et à la diffusion de documents scientifiques de niveau recherche, publiés ou non, émanant des établissements d'enseignement et de recherche français ou étrangers, des laboratoires publics ou privés. 


\title{
INFLUENCE DES RÉGIONS DE CHARGE D'ESPACE SUPERFICIELLES SUR L'ABSORPTION OPTIQUE DES COUCHES MINCES POLYCRISTALLINES DE ZnS
}

\author{
P. BUGNET \\ Laboratoire de Luminescence 1, Faculté des Sciences, Poitiers, France
}

(Reçu le 2 juillet 1973)

\begin{abstract}
Résumé. - Les couches minces polycristallines de $\mathrm{ZnS}$ présentent une absorption optique importante pour les énergies voisines de la largeur de bande interdite. Cette absorption peut être expliquée par un effet analogue à l'effet Franz-Keldysh dans les régions de charge d'espace créées à l'intérieur des grains par les charges superficielles. Un calcul, primitivement établi par Redfield pour les monocristaux, est utilisé pour calculer le potentiel de surface des grains.

Abstract. - Polycristalline $\mathrm{ZnS}$ thin films show an important optical absorption for energies near the band gap. This absorption can be explained by an analog of the Franz-Keldysh effect in the surface charge induced space-charge regions within the grains. An analysis first given by Redfield has been used to obtain the surface potential of the grains.
\end{abstract}

1. Introduction. - Le sulfure de zinc a une largeur de bande interdite voisine de $3,7 \mathrm{eV}$, le maximum de la bande de valence et le minimum de la bande de conduction étant tous deux situés au centre de la première zone de Brillouin. Or il a été constaté depuis longtemps [1], [2] que les couches minces polycristallines de ZnS présentent une queue d'absorption importante, d'allure exponentielle, s'étendant jusque vers $4000 \AA$, alors que le seuil d'absorption bande à bande est situé vers $3350 \AA(3,7 \mathrm{eV})$ pour le cristal parfait. Les transitions indirectes étant peu probables, diverses explications ont été proposées pour cette absorption anormale, faisant appel à des imperfections cristallines ou à des états excités du réseau, mais elles n'expliquent pas les valeurs élevées de la constante d'absorption au voisinage du seuil. Gonella [3] a montré expérimentalement que la dimension des grains de $\mathrm{ZnS}$ était en cause, l'absorption anormale étant surtout importante lorsqu'ils sont petits. Nous avons essayé de la justifier par un effet Franz-Keldysh dans les régions de charge d'espace créées à l'intérieur des grains par des charges superficielles, en utilisant un modèle établi par Redfield [4] pour les monocristaux.

2. Préparation des couches minces. - Les dépôts de $\mathrm{ZnS}$, sur substrat de silice, sont obtenus par pulvérisation cathodique réactive de zinc dans un mélange d'argon et d'hydrogène sulfuré [5]. Les mesures optiques ont porté sur des couches ayant en moyenne
$2000 \AA$ d'épaisseur, préparées dans les conditions suivantes :

- distance anode-cathode : $26 \mathrm{~mm}$,

- tension : $2000 \mathrm{~V}$ continu

- pression totale : $7 \times 10^{-2}$ torr,

- proportion d' $\mathrm{H}_{2} \mathrm{~S}: 16,5 \%$,

- température d'anode : $170^{\circ} \mathrm{C}$,

- température de cathode : $55^{\circ} \mathrm{C}$.

Les dépôts obtenus dans ces conditions ont la structure de la blende.

3. Résultats des mesures d'absorption optique. Le spectre d'absorption est déterminé par des mesures de transmission en incidence normale, le faisceau incident étant produit par une lampe à deutérium et un monochromateur à réseau.

La couche mince de $\mathrm{ZnS}$, d'épaisseur $d$, étant supposée homogène, l'affaiblissement du faisceau est donné par :

$$
I=I_{0} \exp (-\alpha d)
$$

où $\alpha$ est le coefficient d'absorption.

Par suite des réflexions aux interfaces air-ZnS, ZnS-substrat et substrat-air, $\alpha$ n'est pas donné directement par le rapport des intensités transmise et incidente. Cependant, les mesures sont limitées au domaine des absorptions moyennes $\left(10^{3}\right.$ à $\left.10^{5} \mathrm{~cm}^{-1}\right)$ dans lequel l'influence des réflexions multiples peut 
être négligée, avec une erreur inférieure à $10 \%$ dans le cas le plus défavorable, c'est-à-dire lorsque l'absorption est faible [6]. $\alpha$ est alors obtenu en affectant le rapport des intensités transmise et incidente d'un coefficient tenant compte des facteurs de transmission aux divers interfaces. Ceux-ci sont calculés à partir de mesures d'indices réel et imaginaire du $\mathrm{ZnS}$ dues à J. P. Le Bourg [7]. Toutes les mesures ont été effectuées à la température ambiante.

La figure 1 représente l'évolution du coefficient d'absorption $\alpha$ et la figure 2 celle de son carré, en fonction de l'énergie des photons. Pour les énergies supérieures à la largeur de bande interdite, l'absorption suit la loi :

$$
\alpha=K\left(h v-E_{\mathrm{g}}\right)^{1 / 2}
$$

caractéristique des transitions directes permises [8]. Cette courbe extrapolée donne la largeur de bande interdite $E_{\mathbf{g}}$.

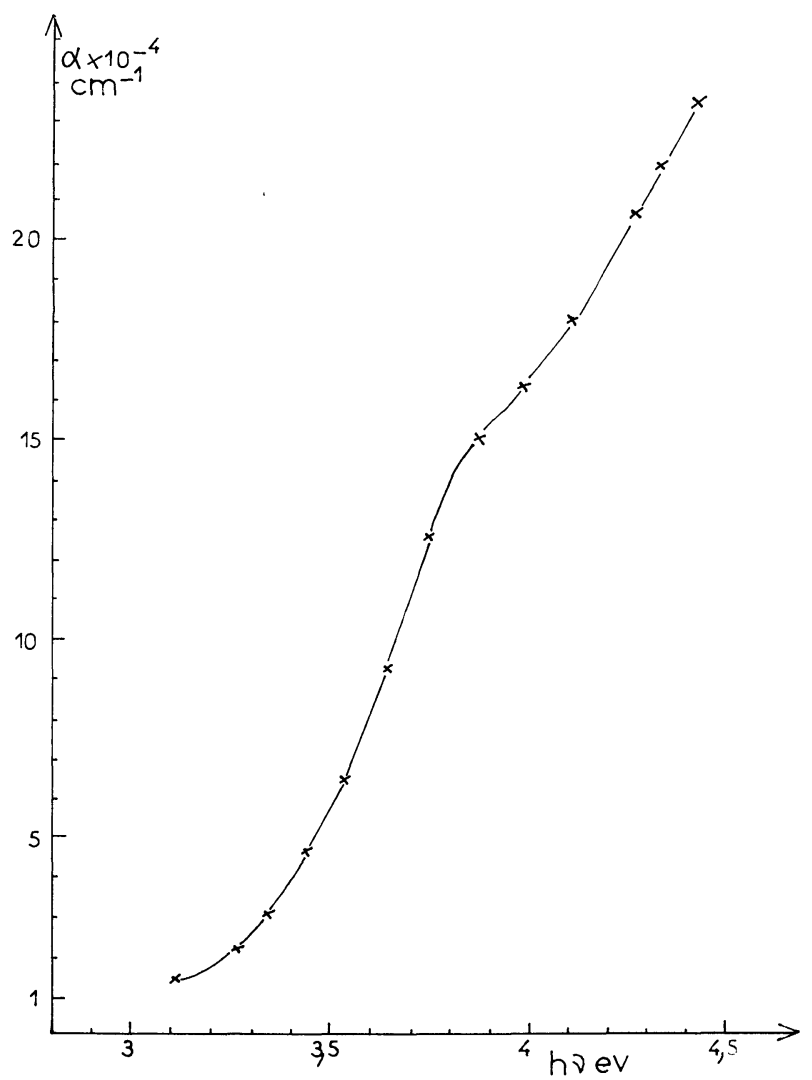

Fig. 1. - Constante d'absorption d'une couche mince de $\mathrm{ZnS}$ en fonction de l'énergie des photons.

A cette absorption bande à bande s'ajoute une absorption supplémentaire, à variation sensiblement exponentielle avec l'énergie (Fig. 3). Au voisinage de la largeur de bande interdite, cette absorption supplémentaire est à peu près indépendante des conditions de préparation (tension inter-électrodes, proportion $\mathrm{d}^{\prime} \mathrm{H}_{2} \mathrm{~S}$ dans le mélange gazeux, températures d'anode et de cathode), donc de la stœchiométrie du $\mathrm{ZnS}$.

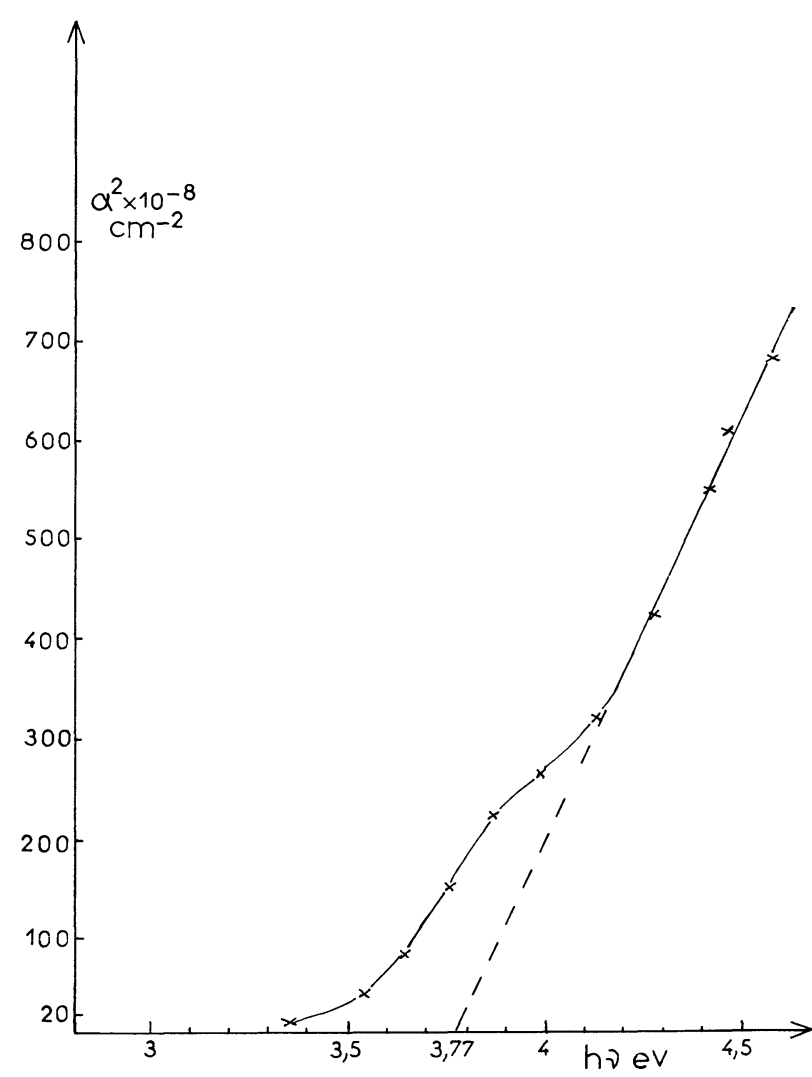

FIG. 2. - Variation du carré de la constante d'absorption avec l'énergie des photons. La partie rectiligne extrapolée donne la largeur de bande interdite.

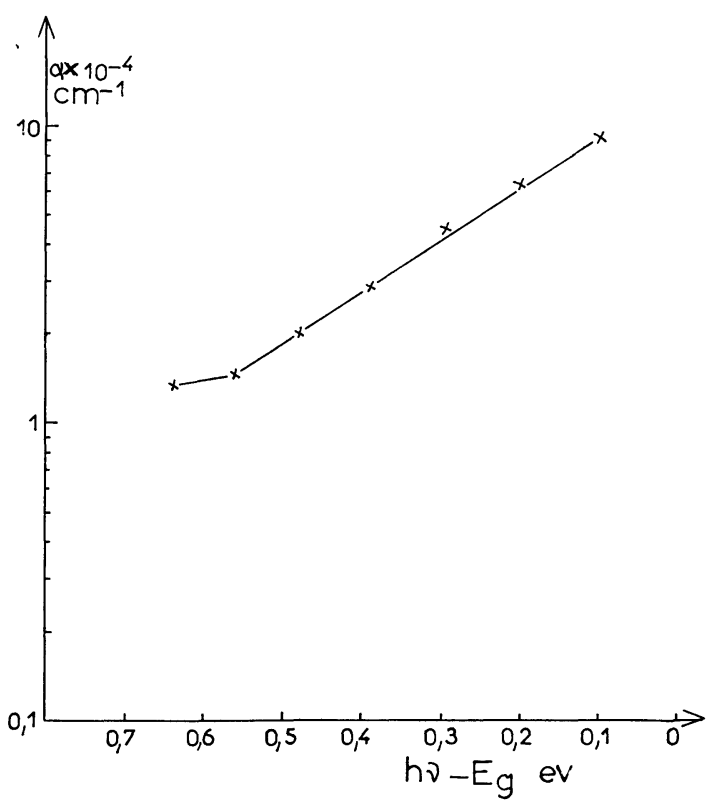

Fig. 3. - Variation de la constante d'absorption avec l'énergie des photons, en dessous de la largeur de bande interdite.

Elle ne dépend pas non plus de l'épaisseur des couches, du moins au-dessus de $1000 \AA$ mais est par contre très sensible à la dimension des grains de sulfure, variant en sens inverse de celle-ci. Il s'agit donc d'un phénomène lié à l'état divisé du $\mathrm{ZnS}$, que son impor- 
tance ne permet cependant pas d'attribuer uniquement à des transitions mettant en jeu des états de surface.

Pour les longueurs d'onde plus grandes, l'absorption supplémentaire dépend fortement des conditions de préparation.

4. Absorption dans les régions de charge d'espace superficielles. - La présence à la surface d'un cristal d'états de surface, tels que les états accepteurs créés par l'oxygène adsorbé sur le $\mathrm{ZnS}$, provoque la formation d'une charge superficielle, et par suite d'une région de charge d'espace à l'intérieur du cristal. Le champ électrique qui règne dans cette région modifie l'absorption bande à bande d'une manière analogue à l'effet Franz-Keldysh. Il existe une telle région de charge d'espace à l'intérieur de chacun des grains constituant la couche polycristalline, de dimensions non négligeables par rapport à celles du grain. La modification de l'absorption bande à bande peut donc être importante et expliquer les anomalies observées.

Etant donnée la complexité que présente l'étude directe d'une couche polycristalline, nous avons essayé d'adapter les calculs effectués sur les monocristaux. Le modèle le plus complet d'absorption dans les régions de charge d'espace superficielle d'un monocristal a été établi par Redfield [4]. Nous rappellerons ses résultats avant de les utiliser pour une couche polycristalline.

4.1 CAS D'UN MONOCRISTAL. - Soit un monocristal d'épaisseur $d$ éclairé par un faisceau de lumière parallèle perpendiculaire à ses faces. Dans les régions de charge d'espace règne un champ électrique $F$, et la constante d'absorption peut s'écrire :

$$
\alpha(\omega)=d^{-1} \int_{0}^{F_{\max }} a(\omega, F) W(F) \mathrm{d} F
$$

où $a$ est une constante d'absorption locale et $W(F)$ le poids statistique du champ électrique $F$. Dans le cas où le cristal est limité par une surface plane on a simplement, en appelant $x$ la coordonnée normale à la surface :

$$
W(F)=\left(\frac{\mathrm{d} F}{\mathrm{~d} x}\right)^{-1} .
$$

Redfield [4] montre que la loi de variation du potentiel dans la région de charge d'espace importe peu, les paramètres importants étant le potentiel de surface $\varphi_{\mathrm{s}}$ et la profondeur de la charge d'espace $\lambda$. Il utilise une variation exponentielle du potentiel :

$$
\varphi=\varphi_{\mathrm{s}} \exp (-x / \lambda)
$$

ce qui entraîne $W(F)=\lambda /|F|$ et établit l'expression suivante du coefficient d'absorption pour la fréquence correspondant à la largeur de bande interdite (unités C. G. S.) :

$\alpha\left(\omega_{\mathrm{g}}\right)=5,4 \times 10^{-4} \pi K\left(\varphi_{\mathrm{s}} \lambda^{2}\right)^{1 / 3} d^{-1}\left(\mathrm{~m} / m_{\mathrm{r}}\right)^{1 / 6} \mathrm{~cm}^{-1}$
$K$ est la pente de la courbe d'absorption fondamentale (formule (1)), $d$ l'épaisseur du cristal, $m$ la masse de l'électron, $m_{\mathrm{r}}$ la masse réduite électron-trou.

4.2 CAS D'une COUChe POlyCRistalline. - Il existe une région de charge d'espace dans chaque grain. Admettons que les grains sont tous sphériques et de même diamètre. Le dépôt est alors constitué par l'empilement de $n$ couches de sphères de diamètre $b$. Or $W(F)$ est le volume occupé par un champ de valeur $F$, à $\mathrm{d} F$ près, par unité de surface de la couche mince. Dans chaque grain sphérique, le volume occupé par un champ de valeur $F$ est donné par :

$$
W(F) \mathrm{d} F=\pi(b-2 x)^{2} \mathrm{~d} x
$$

où $x$ est la distance à la surface du grain. En considérant une file de $n$ grains, dont la surface projetée est $\pi b^{2} / 4$, nous obtenons :

$$
W(F) \mathrm{d} F=4 n W(F) \mathrm{d} F / \pi b^{2}
$$

soit :

$$
W(F)=4 n(1-2 x / b)^{2} \lambda /|F|=4 n \lambda^{\prime}|| F \mid .
$$

$\lambda^{\prime}$ n'est plus comme précédemment une constante, mais ne s'écarte notablement de $\lambda$ que lorsque $x$ n'est pas négligeable devant $b$. Le champ électrique est alors réduit et l'absorption faible, de sorte que l'on ne change pas l'ordre de grandeur du résultat en prenant $\lambda^{\prime}=\lambda$.

Avec $n=d / b$, l'expression (2) devient :

$\alpha\left(\omega_{\mathrm{g}}\right)=2,16 \times 10^{-3} \pi K\left(\varphi_{\mathrm{s}} \lambda^{2}\right)^{1 / 3} b^{-1}\left(m / m_{\mathrm{r}}\right)^{1 / 6} \mathrm{~cm}^{-1}$.

Le coefficient d'absorption est ainsi indépendant de l'épaisseur de la couche mince, mais varie en sens inverse du diamètre des grains, ce qui est conforme aux résultats expérimentaux.

La faible dimension des grains, de l'ordre de $200 \AA$ en moyenne pour les couches étudiées, permet de supposer que la charge d'espace s'étend à tout leur volume. Le rapport $\mathrm{m} / \mathrm{m}_{\mathrm{r}}$ peut d'autre part être pris égal, d'après Cardona [9] à $0,37 \mathrm{~m}$. La relation (3), avec $b=2 \lambda$, donne alors l'expression du potentiel de surface en volts :

$$
\varphi_{\mathrm{s}}=15,6 \alpha^{3}\left(\omega_{\mathrm{g}}\right) K^{-3} \text {. }
$$

Avec les valeurs de $\alpha\left(\omega_{\mathrm{g}}\right)$ et $K$ relevées sur la figure 2 nous obtenons $\varphi_{\mathrm{s}}=1,36 \mathrm{~V}$.

Cette valeur est très élevée, ce qui a déjà été observé sur le ZnS. Swank [10] obtient ainsi, par des mesures de potentiel de contact, une courbure de bandes de l'ordre de $1,2 \mathrm{~V}$.

5. Conclusion. - Il est donc possible d'attribuer l'absorption optique anormale, au niveau du seuil d'absorption fondamentale, à l'effet Franz-Keldysh dans les régions de charge d'espace que créent, à l'intérieur des grains, les charges superficielles. Ce phénomène a également été utilisé par Bujatti-Mar- 
celja [11] pour des couches minces de CdS. Les charges superficielles peuvent être attribuées aux états de surface accepteurs apportés par des atomes d'oxygène adsorbés [12]. L'oxygène figure en effet parmi les gaz résiduels des enceintes à vide, qui comprennent en particulier de la vapeur d'eau. L'hydrogène sulfuré utilisé pour la pulvérisation cathodique réactive en contient également, ainsi que du dioxyde de carbone.

Cependant, cet effet est insuffisant pour expliquer l'absorption aux longueurs d'onde plus grandes. La pente des courbes $\alpha=f(h v)$ que l'on peut calculer à partir de l'effet Franz-Keldysh est en effet très supérieure à celle que l'on observe expérimentalement. Comme nous l'avons déjà mentionné, cette absorption dépend fortement des conditions de préparation, et peut vraisemblablement être attribuée à la présence de défauts ou d'impuretés, notamment entre les grains de $\mathrm{ZnS}$.

\section{Bibliographie}

[1] Coogan, C. K., Proc. Phys. Soc. 70 (1957) 845.

[2] Chavet, L. et Levanone, L., Proc. Phys. Soc. 80 (1962) 1105.

[3] Gonella, J., Symp. Phys. couches minces, Gottingen 1965 (Van den Hoeck, Ruprecht, Gottingen) 1966, p. 280-288.

[4] Redfield, D., Phys. Rev. 140A (1965) 2056.

[5] Batailler, G., Bugnet, P., Deforges, J., Durand, S., C. R. Hebd. Séan. Acad. Sci. Paris 264 (1967) 320.

[6] Bugnet, P., Thèse, Poitiers 1973.
[7] Le Bourg, J. P., Thèse de Docteur Ingénieur, Poitiers 1969.

[8] SмITH, R. A., "Wave mechanics of crystalline Solids" (Chapman et Hall London) 1961.

[9] Cardona, M., J. Phys. Chem. Solids 24 (1963) 1543.

[10] Swank, R. K., Phys. Rev. 153 (1967) 844.

[11] Bujatti, M. et Marcelja, F., Thin solid Films 11 (1972) 249.

[12] Kobayash, A. et Kawaj,, S., J. Chem. Phys. 24 (1956) 907. 